\title{
Versatile Monomer Platform for Plant Oil-Based Latex Coatings
}

\author{
Zoriana Demchuk $^{1}$, Kyle Kingsley ${ }^{1}$, Andriy Voronov ${ }^{1}$ \\ Vasylyna Kirianchuk ${ }^{2}$, Oleg Shevchuk ${ }^{2}$, Ananiy Kohut ${ }^{2}$, Stanislav Voronov ${ }^{2}$ \\ ${ }^{1}$ North Dakota State University, Dept. 2760 \\ PO Box 6050, Fargo, ND, USA \\ andriy.voronov@ndsu.edu \\ ${ }^{2}$ Lviv Polytechnic National University, Department of Organic Chemistry \\ 12 Bandera Street, Lviv, Ukraine \\ stanislav.voronov@gmail.com
}

\section{Extended Abstract}

Today coatings made from renewable biobased resources are still being over performed by coatings made from petroleum-based sources in the way they act mechanically, thus leaving them less useful from the industrial standpoint. Such properties of films/coatings as toughness or rigidness are accessible for control through regulating their cross-link density. As the curing process continues, the double bonds that do not get polymerized are capable of reforming to link the alkyl groups of monomers together, thus varying the cross-link density of the material. Use of plant-oil-based monomers in this case can be advantageous because the amount of unsaturation of the fatty acid chains in those monomers is generally much higher than that of their petro-based counterparts and can be regulated.

Using the developed in our group one-step method of plant oil direct transesterification, a library of acrylic monomers has been recently synthesized [1-4]. Using emulsion and miniemulsion polymerization latexes from new monomers based on plant oils (soybean, linseed, canola, sunflower, high oleic soybean, olive, hydrogenated soybean oil) (POBMs) with remarkably different amount of unsaturation (compositions of fatty acids in oil triglycerides) have been developed.

Presence of plant oil-based fragments impacts thermomechanical properties of latex films by decreasing glass transition temperature (thus, providing plasticizing effect) of latex copolymers. The thermomechanical properties of resulting latex nanoparticles depend considerably on the amount of incorporate oil-derived fragments. Biobased ingredient makes latex copolymers more flexible, improves material film forming properties, provides some flexibility and toughness, as compared to the normally rigid polystyrene and poly(methyl methacrylate). In addition, plant oil-based fragments enhance hydrophobicity of the resulting latex films and can be considered as additives to reduce water sensitivity of the polymer.

At this point, stable latexes containing up to $70 \mathrm{wt} \%$ of biobased content can be synthesized using the miniemulsion process of copolymerization of POBMs with different vinyl counterparts. Their unsaturation amount can be utilized as a criterion in studying the resulting latex properties, including the effect of variation in monomer feed unsaturation on the crosslink density of latex films and, consequently, on polymer thermal and mechanical properties. It is observed that in oxidatively cured latex films, the crosslink density depends linearly on reaction feed unsaturation. Based on that, the latex network thermomechanical properties can be adjusted by simply combining various plant oil-based monomers at certain ratios ("given" unsaturations) in the reaction feed.

\section{References}

[1] I. Tarnavchyk, A. Popadyuk, N. Popadyuk, and A. Voronov, "Synthesis and Free Radical Copolymerization of Vinyl Monomer from Soybean Oil,” ACS Sustainable Chemistry \& Engineering, vol. 3, no. 7, pp. 1618-1622, 2015.

[2] Z. Demchuk, O. Shevchuk, I. Tarnavchyk, V. Kirianchuk, A. Kohut, S. Voronov, and A. Voronov, "Free Radical Polymerization Behavior of the Vinyl Monomers from Plant Oil Triglycerides," ACS Sustainable Chemistry \& Engineering, vol. 4, no. 12, pp. 6974-6980, 2016.

[3] Z. Demchuk, O. Shevchuk, I. Tarnavchyk, V. Kirinachuk, M. Lorenson, A. Kohut, S. Voronov, A. Voronov, "Free Radical Copolymerization Behavior of Plant Oil-Based Vinly Monomers and Their Feasibility in Latex Synthesis," ACS Omega, vol. 1, no. 6, pp. 1374-1382, 2016.

[4] K. Kingsley, O. Shevchuk, S. Voronov, and A. Voronov, "Features of Emulsion Copolymerization for Plant Oil-Based Vinyl Monomers and Styrene," Industrial Crops and Products, vol. 109, pp. 274-280, 2017. 